\title{
MicroRNA-663b downregulation inhibits proliferation and induces apoptosis in bladder cancer cells by targeting TUSC2
}

\author{
JIANYING CHEN* ${ }^{*}$, BO SONG ${ }^{*}$ and GUANGQI KONG \\ Urology Department, Beijing Luhe Hospital, Capital Medical University, Beijing 101149, P.R. China
}

Received May 23, 2018; Accepted January 25, 2019

DOI: $10.3892 / \mathrm{mmr} .2019 .10023$

\begin{abstract}
The present study aimed to explore the role and underlying molecular mechanism of microRNA-663b (miR-663b) in the tumorigenesis of bladder cancer. The miR-663b expression in human bladder cancer tissues and cell lines was measured determined reverse transcription-quantitative polymerase chain reaction. TargetScan was used to predict the potential targets of miR-663b, and a dual-luciferase reporter assay was performed to validate tumor suppressor candidate 2 (TUSC2) as a target of miR-663b. Cell Counting Kit-8 was used for cell viability analysis, and cell apoptosis was evaluated by flow cytometry. In addition, western blot analysis was performed to detect protein expression in current study. The findings suggested that miR-663b was upregulated in bladder cancer tissues and cell lines compared with normal tissue and cells. TUSC 2 was validated as a direct target of miR-663b and was negatively regulated by miR-663b. miR-663b inhibition significantly reduced the viability of T24 cells, and T24 cell apoptosis was markedly induced. In addition, miR-663b inhibition enhanced the expression levels of p53 and p21 in T24 cells. Furthermore, the changes caused by miR-663b inhibitor in T24 cells were eliminated by TUSC 2 gene silencing. In conclusion, inhibition of miR-663b reduced viability and induced apoptosis in bladder cancer cells by targeting TUSC2. These findings provide a promising novel therapeutic target for bladder cancer treatment.
\end{abstract}

\section{Introduction}

Bladder cancer is one of the most common urological tumors. The incidence of bladder cancer ranks as the ninth most common malignant tumor worldwide, and a large number of

Correspondence to: Dr Guangqi Kong, Urology Department, Beijing Luhe Hospital, Capital Medical University, 82 Xinhua South Road, Tongzhou, Beijing 101149, P.R. China

E-mail: konggq11@163.com

*Contributed equally

Key words: bladder cancer, microRNA-663b, tumor suppressor candidate 2, proliferation, apoptosis new cases re diagnosed every year $(1,2)$. In China, bladder cancer is the most common malignant tumor of the urinary system, and $>90 \%$ bladder cancer is transitional cell carcinoma with a high recurrence rate and invasive ability (3). In recent years, the incidence of bladder cancer in some cities in China has had a steady upward trend. At present, the predominant treatment methods for bladder cancer are surgical resection, radiotherapy and chemotherapy. The higher recurrence rate and tumor progression rate still greatly impact the prognosis of patients with bladder cancer. Radical cystectomy can effectively reduce the recurrence rate of patients with bladder cancer, improve their long-term survival rate and is the standard treatment method for invasive bladder cancer (4). However, surgical treatment can seriously affect the quality of life of patients $(5,6)$. Therefore, it is necessary to provide a quality transitional care service (7) to ensure the smooth transition of patients with bladder cancer from hospital to home, and to improve the lack of professional guidance available for home rehabilitation, to meet the needs of patients and improve the quality of life and prognosis of the patients. Currently, the transitional care service of China is still in its infancy, a lack of continuity and coordination of nursing activities for patients with bladder cancer. Therefore, the search for novel and effective strategies for the treatment of bladder cancer is urgent and is of major clinical significance.

MicroRNAs (miRNAs), a family of small non-coding single-stranded RNAs (20-22 nucleotides in length), have a critical role in post-transcriptionally regulating gene expression by binding to the $3^{\prime}$ untranslated region (3'UTR) of target genes (8-10). Studies demonstrated that abnormal miRNA expression is associated with the occurrence of numerous types of tumor, including bladder cancer (11-13). miRNAs can act as oncogenes or tumor suppressors, and the altering abnormal expression of an miRNA can inhibit tumorigenesis and progression (14). With the status and role of miRNA in tumor research attracting increasing attention, research on miRNA and bladder cancer has also developed and deepened.

A previous study reported that miRNA-663b (miR-663b) was highly expressed in the plasma of patients with bladder cancer, and may be a promising novel circulating biomarker for the clinical detection of bladder cancer (15). However, the expression of miR-663b in the tumor tissues of bladder cancer patients and its specific role remain unclear.

Numerous previous studies demonstrated that tumor suppressor 2, mitochondrial calcium regulator (TUSC2) is 
a tumor suppressor gene $(16,17)$. TUSC2 was identified to induce G1 phase cell cycle arrest and apoptosis (18), to regulate calcium signaling (19) and tyrosine kinase activity (20), and to affect gene expression (21). TUSC2 is associated with various signaling pathways, including the p53 pathway (22). However, to the best of the authors' knowledge, the association between miR-663b and TUSC 2 in bladder cancer cells has not been previously investigated.

Therefore, the aim of the current study was to investigate the expression and specific role of miR-663b in bladder cancer, and further to explore the associated mechanisms. The findings may help to provide novel and effective targets, and an additional theoretical basis for the treatment of bladder cancer.

\section{Materials and methods}

Clinical samples. A total of 25 paired bladder cancer tissues and the adjacent normal tissues were obtained from 25 patients with bladder cancer (18 male, 7 female; age range, 27-61 years old) at the Beijing Luhe Hospital (Beijing, China) between May 2015 and May 2017. Informed consent was obtained from every patient, and the study was approved by The Ethics Committee of Beijing Luhe Hospital.

Cell culture and cell transfection. Bladder cancer cell lines T24, 5637, J82, UMUC3, and a normal uroepithelium cell line (SV-HUC-1) were obtained from the Shanghai Institute of Cell Biology, Chinese Academy of Sciences (Shanghai, China). The cell lines were cultured in Dulbecco's Modified Eagle's Medium (Invitrogen; Thermo Fisher Scientific, Inc., Waltham, MA, USA) supplement with $10 \%$ fetal bovine serum (Sigma-Aldrich; Merck KGaA, USA) and 1\% streptomycin-penicillin mix solution, and incubated at $37^{\circ} \mathrm{C}$ with $5 \%$ $\mathrm{CO}_{2}$.

T2 4 cells were seeded in 6-well plates at a density of $1 \times 10^{6}$ cells/well and cultured at $37^{\circ} \mathrm{C}$ for $24 \mathrm{~h}$. T2 4 cells were transfected with $100 \mathrm{nM}$ miR-663b inhibitor (cat. no. CIH0743; Guangzhou Weijia Technology Co., Ltd., Guangzhou, China), 100 nM negative control (NC; cat. no. CS8005; Biomics Biotechnologies Co., Ltd., Nantong, China), $10 \mu \mathrm{M}$ control-small interfering RNA (siRNA; cat. no. sc-36869; Santa Cruz Biotechnology, Inc., Dallas, TX, USA), $10 \mu \mathrm{M}$ TUSC2-siRNA (cat. no. sc-77910; Santa Cruz Biotechnology, Inc.), or miR-663b inhibitor + TUSC2-siRNA at $37^{\circ} \mathrm{C}$ for $48 \mathrm{~h}$ by using Lipofectamine ${ }^{\circledR} 2000$ (Thermo Fisher Scientific, Inc.) according to the manufacturer's instruction. The working concentration was determined following a dose-response analysis (data not shown). Cells without any treatment were used as the control. Subsequent analyses were performed at $48 \mathrm{~h}$ after cell transfection.

Dual luciferase reporter assay. A bioinformatics software (TargetScan; version 7.2; http://www.targetscan.org/vert_72/) was used to predict the potential targets of miR-663b, and an miR-663b target site was identified in the TUSC2 3'UTR. To confirm this prediction, a dual luciferase reporter assay was performed. Wild-type (WT; 5'-AGGAGCCCAGGC UGGGGCCACA-3') and mutant (MUT; 5'-AGGAGCCCA GGCUGAUAUAGAA-3') TUSC2 3'UTRs were synthesized using the PrimeScript reverse transcription (RT) reagent kit
(Takara Bio, Inc., Otsu, Japan) according to the manufacturer's protocol, using RNA extracted from T24 cells. The reverse transcription was performed by incubating the reaction at $50^{\circ} \mathrm{C}$ for $15 \mathrm{~min}$ and at $85^{\circ} \mathrm{C}$ for $2 \mathrm{~min}$. The cDNA obtained was subsequently used as template to clone the $3^{\prime} \mathrm{UTR}$ into the psiCHECK-2 reporter. Point mutations in the binding site for miR-663b in the 3'UTR of TUSC2 were generated using the QuikChange Site-Directed Mutagenesis kit (Stratagene; Agilent Technologies, Inc., Santa Clara, CA, USA) according to the manufacturer's protocol. miR-663b mimic (cat. no. HmiR-SN0775-SN; GeneCopoeia, Inc., Rockville, MD, USA) at a concentration of $50 \mathrm{nM}$ or mimic control (cat. no. CmiR-SN0001-SN; GeneCopoeia, Inc.) at a concentration at $50 \mathrm{nM}$ and $10 \mu \mathrm{l} \mathrm{miR-663b-TUSC2-WT} \mathrm{3'UTR} \mathrm{or} 10 \mu \mathrm{l}$ miR-663b-TUSC2-MUT 3'UTR vector were co-transfected into T24 cells $\left(5 \times 10^{4}\right.$ cells/well) using Lipofectamine ${ }^{\circledR} 2000$ (Thermo Fisher Scientific, Inc.) according to the manufacturer's protocol. At $48 \mathrm{~h}$ after the transfection, the luciferase activity was analyzed by performing the dual-luciferase reporter assay system (Promega Corporation, Madison, WI, USA) according to the manufacturer's instruction, and normalized to Renilla luciferase activity.

Cell viability assay. At $48 \mathrm{~h}$ following transfection, a Cell Counting Kit-8 (CCK-8) (cat. no. C0038; Beyotime Institute of Biotechnology, Haimen, China) assay was performed to detect cell viability ability. In brief, T24 cells were collected, re-suspended and then re-plated into 96-well culture plates ( $5 \times 10^{3}$ cells/well) (Corning Incorporated, Corning, NY, USA). The cells were incubated for $24 \mathrm{~h}$ at $37^{\circ} \mathrm{C}$ with $5 \% \mathrm{CO}_{2}$. Subsequently, CCK-8 solution $(10 \mu \mathrm{g} / \mathrm{ml})$ was added to each well, and then incubated at $37^{\circ} \mathrm{C}$ for $2 \mathrm{~h}$. Finally, the optical density value at $450 \mathrm{~nm}$ was determined using a microplate reader (Thermo Fisher Scientific, Inc.). Each experiment was repeated at least three times.

Apoptosis analysis assay. At $48 \mathrm{~h}$ after cell transfection, cell apoptosis analysis was performed. The transfected T24 cells were washed with PBS, labeled with $5 \mu$ l Annexin $\mathrm{V}$-fluorescein isothiocyanate and $5 \mu \mathrm{l}$ propidium iodide with the Annexin V-FITC Apoptosis Detection kit (cat. no. C1062; Beyotime Institute of Biotechnology), and then incubated at room temperature for $30 \mathrm{~min}$ in dark. A flow cytometer (BD Biosciences, Franklin Lakes, NJ, USA) was used to analyze cell apoptosis, and the proportion of apoptotic cells was calculated. Experiments were repeated at least three times.

Western blot analysis. At $48 \mathrm{~h}$ after cell transfection, western blotting was used to detect protein expression. Radioimmunoprecipitation assay buffer (Auragene Bioscience, Changsha, China) was used to extract the total cellular proteins. A bicinchoninic acid protein quantitative kit (Thermo Fisher Scientific, Inc.) was performed to detect protein concentration following the manufacturer's instructions. Protein samples (25 $\mu \mathrm{g} /$ lane) were separated by using SDS-PAGE on $11 \%$ gels and then transferred onto polyvinylidene fluoride membranes. Following blocking with 5\% skim milk at room temperature for $1 \mathrm{~h}$, the membranes were then incubated with a primary antibody: Anti-TUSC2 (cat. no. sc-517369; 1:1,000; Santa Cruz Biotechnology, Inc.), anti-p53 (cat. no. 2527; 1:1,000; 
Cell Signaling Technology, Inc., Danvers, MA, USA), anti-p21 (cat. no. 2947; 1:1,000; Cell Signaling Technology, Inc.), anti- $\beta$-actin(cat. no. 4970; 1:2,000; Cell Signaling Technology, Inc.) at $4^{\circ} \mathrm{C}$ overnight. Subsequently, the membranes were washed with TBS-Tween solution three times, then the membranes were incubated with an anti-rabbit immunoglobulin $\mathrm{G}$ horseradish peroxidase-conjugated secondary antibody (1:5,000; cat no. 7074; Cell Signaling Technology, Inc.) at room temperature for $4 \mathrm{~h}$. Finally, the protein bands were visualized using an enhanced chemiluminescence kit (Applygen Technologies, Inc., Beijing, China) according to the manufacturer's protocol.

$R T$-quantitative polymerase chain reaction (qPCR). Total RNA from tissues and cells was extracted by using TRIzol ${ }^{\circledR}$ reagent (Invitrogen; Thermo Fisher Scientific, Inc.) following the manufacturer's instructions. Absorbance 260/280 ratio was calculated to assess the integrity and quality of the RNA. U6 (for miRNA) and GAPDH (for mRNA) were used as the internal controls. Total RNA was reverse transcribed into cDNA using the TaqMan microRNA RT kit (Thermo Fisher Scientific, Inc.) according to the manufacturer's instructions. mRNA was reverse transcribed into cDNAs using the PrimeScript RT reagent kit (Takara Bio, Inc.) according to the manufacturer's protocol. The reverse transcription was performed by incubating the reaction at $50^{\circ} \mathrm{C}$ for $15 \mathrm{~min}$ and at $85^{\circ} \mathrm{C}$ for $2 \mathrm{~min}$. RT-qPCR was performed using SYBR Premix Ex Taq (Takara Bio, Inc.) according to the manufacturer's instructions. The thermocycling conditions were as follows: Initial denaturation at $95^{\circ} \mathrm{C}$ for $5 \mathrm{~min}$, followed by 40 cycles of denaturation at $95^{\circ} \mathrm{C}$ for $15 \mathrm{sec}$ and annealing/elongation at $60^{\circ} \mathrm{C}$ for $30 \mathrm{sec}$. The primer sequences used for PCR were as follows: GAPDH, forward 5'CTTTGGTATCGTGGAAGGACTC3', reverse 5'GTAGAG GCAGGGATGATGTTCT3'; U6, forward 5'GCTTCGGCA GCACATATACTAAAAT3', reverse 5'CGCTTCACGAAT TTGCGTGTCAT3'; TUSC2, forward 5'GGAGACAATCGT CACCAAGAAC3', reverse 5'TCACACCTCATAGAGGAT CACAG3'; p53, forward 5'CTGCCCTCAACAAGATGT TTTG3', reverse 5'CTATCTGAGCAGCGCTCATGG3'; p21, forward 5'ATGAAATTCACCCCCTTTCC3', reverse 5'CCC TAGGCTGTGCTCACTTC3'; miR-663b, forward 5'CAT AATAAATAGGCGGGGCG3', reverse 5'CAGAGCAGGG TCCGAGGTA3'. The relative gene expression was determined using the $2^{-\Delta \Delta C q}$ method (23).

Statistical analysis. SPSS 16.0 statistical software (SPSS, Inc., Chicago, IL, USA) was used for statistical analyses. Data are presented as the mean \pm standard deviation. Student's t-test or one-way analysis of variance followed by Tukey's test was performed to compare differences between groups. $\mathrm{P}<0.05$ was considered to indicate a statistically significant difference.

\section{Results}

miR-663b is upregulated in bladder cancer. RT-qPCR was performed to detect the level of miR-663b in bladder cancer tissues and cell lines (T24, 5637, J82, UMUC3) and the normal bladder cell line (SV-HUC-1). Compared with adjacent normal tissues, the level of miR-663b was significantly increased in bladder cancer tissues (Fig. 1A). Additionally, bladder cancer cell lines (T24, 5637, J82, UMUC3) exhibited higher expression of miR-663b than the normal bladder cell line (SV-HUC-1). miR-663b was highest in T24 cells (Fig. 1B). The data indicated that miR-663b was upregulated in bladder cancer. T24 cells were used for subsequent analysis.

TUSC2 is a target of $m i R-663 b$. To study the precise role of miR-663b in bladder cancer, bioinformatics software (TargetScan) we used to predict the potential targets of miR-663b, and TUSC 2 was revealed to have an miR-663b binding site in the 3'UTR (Fig. 2A). The luciferase reporter gene assay confirmed that miR-663b binds the TUSC2 3'UTR to reduce TUSC2 expression (Fig. 2B).

Furthermore, the level of TUSC2 was detected in bladder cancer cell line T24 and the normal bladder cell line SV-HUC-1, and the findings suggested TUSC2 was significantly down-regulated in T24 cells at the protein and mRNA level compared with the SV-HUC-1 cells (Fig. 3A and B). To analyze the role of miR-663b, T24 cells were transfected with miR-663b inhibitor, a negative control, control-siRNA, TUSC2-siRNA, or miR-663b inhibitor + TUSC2-siRNA for $48 \mathrm{~h}$. The transfection efficiency of miR-663b inhibitor was evaluated using RT-qPCR, and the results indicated that miR-663b inhibitor significantly decreased the level of miR-663b in T24 cells (Fig. 3C). TUSC2-siRNA significantly reduced the mRNA and protein level of TUSC2 in T24 cells (Fig. 3D and E). miR-663b inhibitor markedly enhanced the expression level of TUSC2, and this increase was eliminated by TUSC2-siRNA (Fig. 3F and G).

miR-663b inhibitor reduced T24 cell viability. A CCK-8 assay was used to evaluate the effect of miR-663b on T24 cell proliferation. miR-663b inhibitor significantly reduced T24 cell viability, which was eliminated by TUSC2-siRNA (Fig. 4).

miR-663b inhibitor induced T24 cell apoptosis. The effect of miR-663b on T24 cell apoptosis was analyzed by using flow cytometry. The findings suggested that compared with the control group, miR-663b inhibition significantly induced T24 cell apoptosis, and this increase was reversed by TUSC2-siRNA (Fig. 5A and B).

miR-663b down-regulation enhanced the expression of $p 53$ and $p 21$. A previous study suggested that miR-663b down-regulation promoted $\mathrm{p} 53$ and $\mathrm{p} 21$ expression in osteosarcoma cells (24). Additionally, TUSC 2 has been revealed to be associated with the p53 pathway (22). Therefore, to explore the underlying molecular mechanism of the role of miR-663b in T2 4 cells, expression of p53 and p21 was detected by western blot analysis and RT-qPCR. Compared with the control group, the protein expression levels of p53 and p21 were notably enhanced by miR-663b inhibitor, and these effects were eliminated by TUSC2-siRNA (Fig. 6A). The same trend was detected by RT-qPCR (Fig. 6B and C).

\section{Discussion}

Bladder cancer is one of the most common malignant tumors in humans, with a complex pathogenesis involving numerous 
A

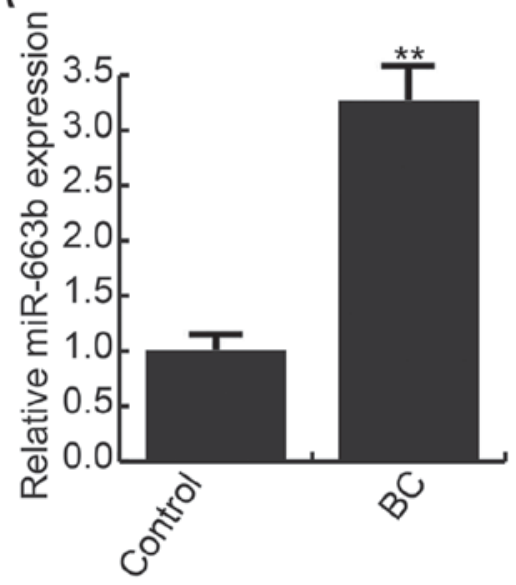

B

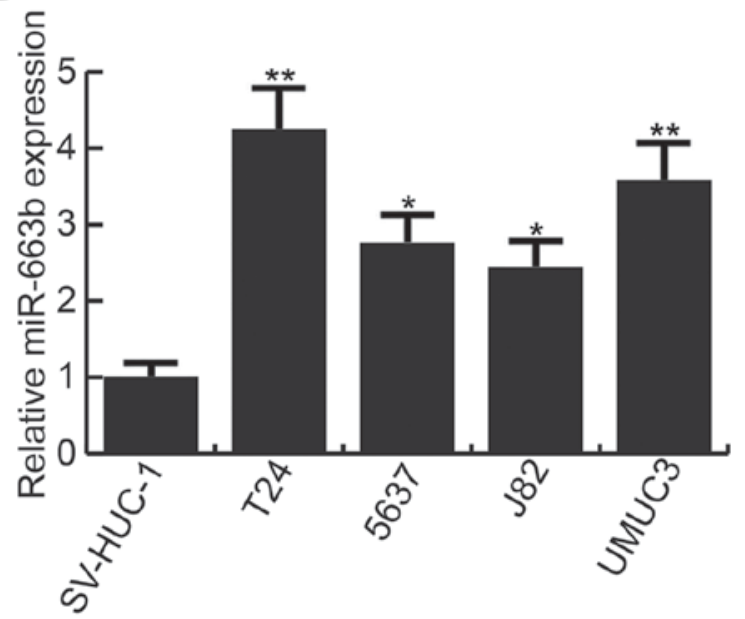

Figure 1. miR-663b is up-regulated in bladder cancer. Reverse transcription-quantitative polymerase chain reaction was used to determine miR-663b expression. (A) Relative miR-663b expression in BC tissues. (B) Relative miR-663b expression in bladder cancer cell lines. SV-HUC-1, the normal bladder cell line; T24, 5637, J82, UMUC3, human bladder cancer cell lines. ${ }^{*} \mathrm{P}<0.05,{ }^{* *} \mathrm{P}<0.01$ vs. control or SV-HUC-1. Experiments were repeated three times. miR, microRNA; BC, bladder cancer tissues; Control, adjacent normal tissues.

A

\begin{tabular}{|c|c|c|}
\hline $\begin{array}{l}\text { Position } 93-100 \text { of TUSC2 } 3 \text { UTR } \\
\text { hsa-miR- } 663 \mathrm{~b}\end{array}$ & $5^{\prime}$ & $\begin{array}{l}\text { ‥ GAGGAGCCCAGGCUGGGGCCACA. . } \\
\text { IIIIIII } \\
\text { GGAGUCCGUGCCGGCCCGGUGG }\end{array}$ \\
\hline
\end{tabular}

B

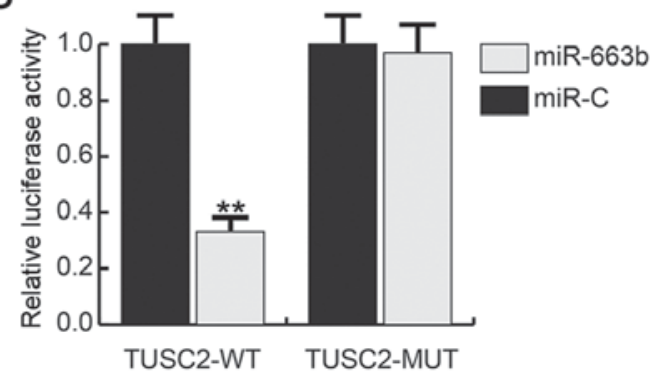

Figure 2. TUSC2 is a direct target of miR-663b. (A) Interaction between miR-663b and 3'UTR of TUSC2 was predicted TargetScan. (B) Luciferase activity of a reporter containing wild-type TUSC2 3'UTR or a mutant TUSC2 $3^{\prime} \mathrm{UTR}$. ${ }^{* *} \mathrm{P}<0.01$ vs. control. Experiments were repeated three times. TUSC2, tumor suppressor candidate 2; UTR, untranslated region; miR, microRNA; miR-C, mimic control; WT, wild-type; MUT, mutant.

genes, aberrant protein functions and changes in multiple signaling pathways. Currently, the molecular genetics of the development and progression of bladder cancer remain unclear. In recent years, the pathological relevance and importance of miRNAs in bladder cancer have attracted increasing attention. In the present study, the expression, biological function and molecular mechanisms of miR-663b and its target gene in the pathogenesis of bladder cancer were investigated.

miR-663b inhibition reduced bladder cancer cell viability and induced cell apoptosis. miR-663b was demonstrated to directly target TUSC 2 and negatively regulated TUSC2 expression. miR-663b inhibition indirectly promoted the expression of p53 and p21, which may contribute to the prevention of T24 cell proliferation and the induction of T24 cell apoptosis. miR-663b in bladder cancer tissues and cell lines, compared with normal tissue and a normal bladder cell line, respectively. T24 cells had the highest miR-663b level of the cell lines. Therefore, in the current study, T24 cells were used for the investigation of bladder cancer in vitro. A novel functional link between miR-663b and TUSC2 in the tumorigenesis of bladder cancer was also revealed. miR-663b inhibitor significantly reduced cell viability and increased apoptosis of T24 cells in vitro. miR-663b inhibition significantly enhanced the expression levels of p53 and p21. Furthermore, the effects of miR-663b inhibitor in T24 cells were eliminated by TUSC2-siRNA. These results indicated that miR-663b acts as an oncogene in the development of bladder cancer, and miR-663b inhibitor had anti-tumor activity; thus, miR-663b may be as a promising therapeutic target for bladder cancer treatment.

Various studies suggested that miRNAs have critical roles in the diagnosis, therapy and prognosis of a variety of cancers and can be involved in tumorigenesis, tumor growth and tumor metastasis $(25,26)$. Therefore, miRNAs may be promising treatment targets for cancer. Increasing evidence has indicated that numerous miRNAs are involved in bladder cancer cell proliferation, migration and invasion (27-31). High expression of miR-663b has been reported in the plasma of patients with bladder cancer (15); however, to the best of our knowledge, the expression of miR-663b in bladder tumors and its specific role remain unclear. He findings of the current demonstrated that miR-663b was significantly upregulated in bladder cancer tissues and cell lines compared with adjacent tissues and normal bladder cells, and miR-663b inhibitor markedly repressed the viability of T24 cells and induced apoptosis.

TUSC2 is a recognized tumor suppressor gene $(16,17)$. A variety of allele losses and genetic alterations are observed in various cancers, including breast cancer, lung cancer and others $(17,32,33)$. Studies have reported that TUSC2 can induce G1 phase cell cycle arrest and apoptosis (18), regulate calcium signaling (19) and tyrosine kinase activity (20), and affect gene expression (21). TUSC2 is associated with several pathways, including the p53 pathway (22). However, to the 
A

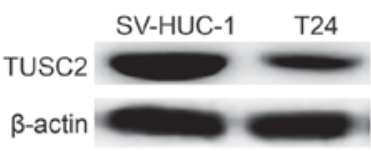

B

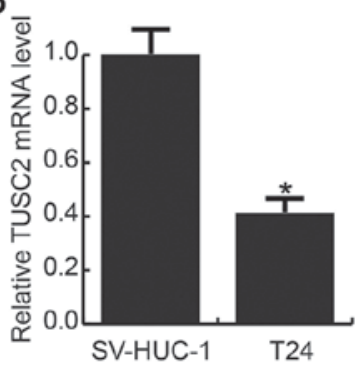

C

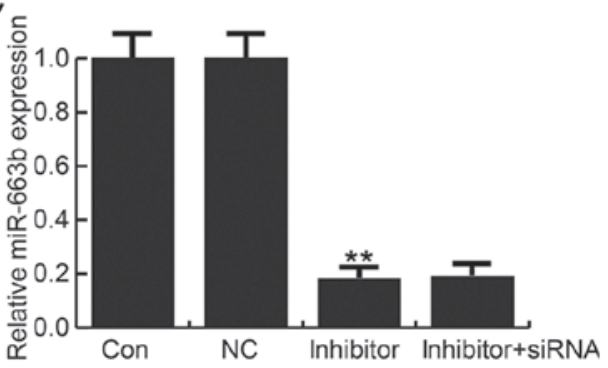

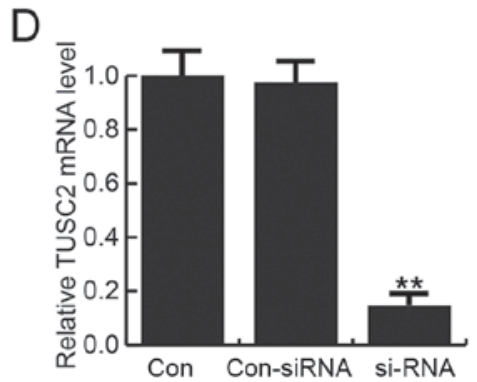

E

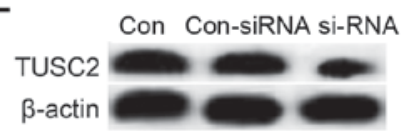

F

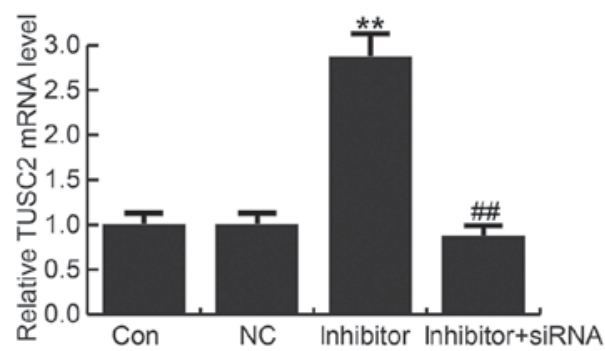

G

Con NC inhibitor inhibitor+siRNA

\section{TUSC2}

$\beta$-actin

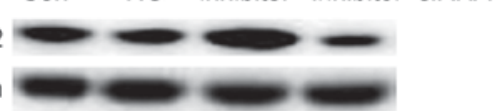

Figure 3. Effect of miR-663b inhibitor and TUSC2 siRNA on expression of TUSC2. Reverse transcription-quantitative polymerase chain reaction was used to determine the miR-663b and TUSC2 mRNA expression, and western blotting was used for protein level detection. (A) Protein level of TUSC2 in SV-HUC-1 and T24 cells. (B) mRNA level of TUSC2 in SV-HUC-1 and T24 cells. (C) Relative miR-663b expression in T24 cells transfected with miR-663b inhibitor and TUSC2 siRNA. (D) mRNA and (E) protein level of TUSC2 in T24 cells transfected with TUSC2 siRNA. (F) mRNA and (G) protein level of TUSC2 in T24 cells transfected with miR-663b inhibitor and TUSC2 siRNA. All data are presented as the mean \pm standard deviation of three independent experiments. ${ }^{* *} \mathrm{P}<0.01$ vs. control; ${ }^{\# \#} \mathrm{P}<0.01$ vs. inhibitor. TUSC2, tumor suppressor candidate 2; miR, microRNA; Con, control group, no treatment; NC, negative control inhibitor; siRNA, TUSC2-small interfering RNA.

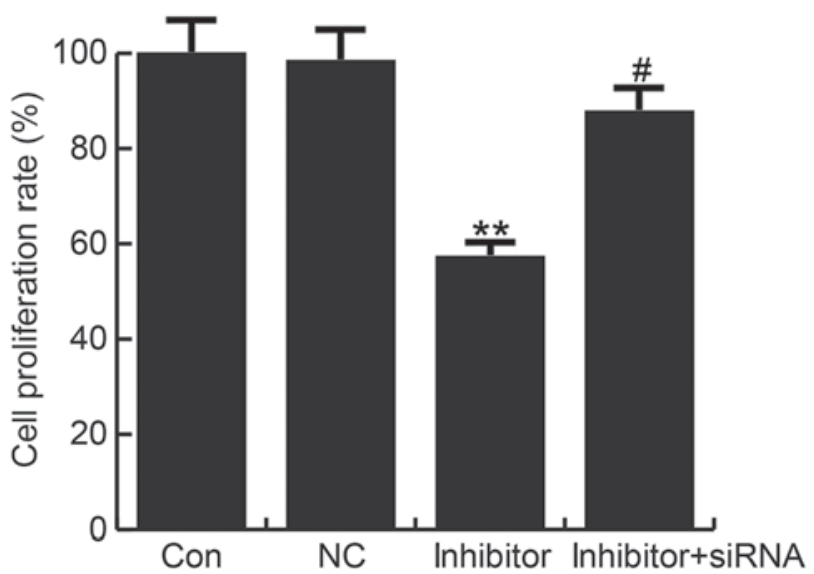

Figure 4. miR-663b inhibition reduces the proliferation of T24 cells. Effect of miR-663b inhibitor and TUSC2-siRNA on cell proliferation was analyzed using Cell Counting Kit- 8 assay in T24 cells. All data are presented as the mean \pm standard deviation of three independent experiments. ${ }^{* *} \mathrm{P}<0.01$ vs. control; ${ }^{\text {P }}<0.01$ vs. inhibitor. miR, microRNA; TUSC2, tumor suppressor candidate 2; Con, control group, no treatment; $\mathrm{NC}$, negative control inhibitor; siRNA, TUSC2-small interfering RNA. best of our knowledge, no interaction between miR-663b and TUSC2 in bladder cancer cells has been reported previously. In the present study, TUSC2 was identified as a direct target of miR-663b, and it was demonstrated that miR-663b affects bladder cancer cell viability and apoptosis by directly targeting TUSC2. Additionally, as TUSC2 is associated with p53 pathway (22), and it was speculated as to whether the effect of miR-663b on bladder cancer cells was associated with p53 pathway, thus p53 and p21 expression was analyzed in the current study. The results indicated that miR-663b inhibition increased the expression of p53 and p 21 in bladder cancer cells.

In summary, the current study demonstrated that miR-663b was upregulated in bladder cancer tissues and cell lines, and inhibition of miR- $663 \mathrm{~b}$ reduced the viability of bladder cancer cells, induced cell apoptosis and enhanced the expression of p53 pathway proteins by directly targeting TUSC 2 . Therefore, miR-663b may serve as a promising therapeutic target for the treatment of bladder cancer. However, this is a preliminary study of the role of miR-663b in bladder cancer, and further research is required in order to make the role of miR-663b in 
A

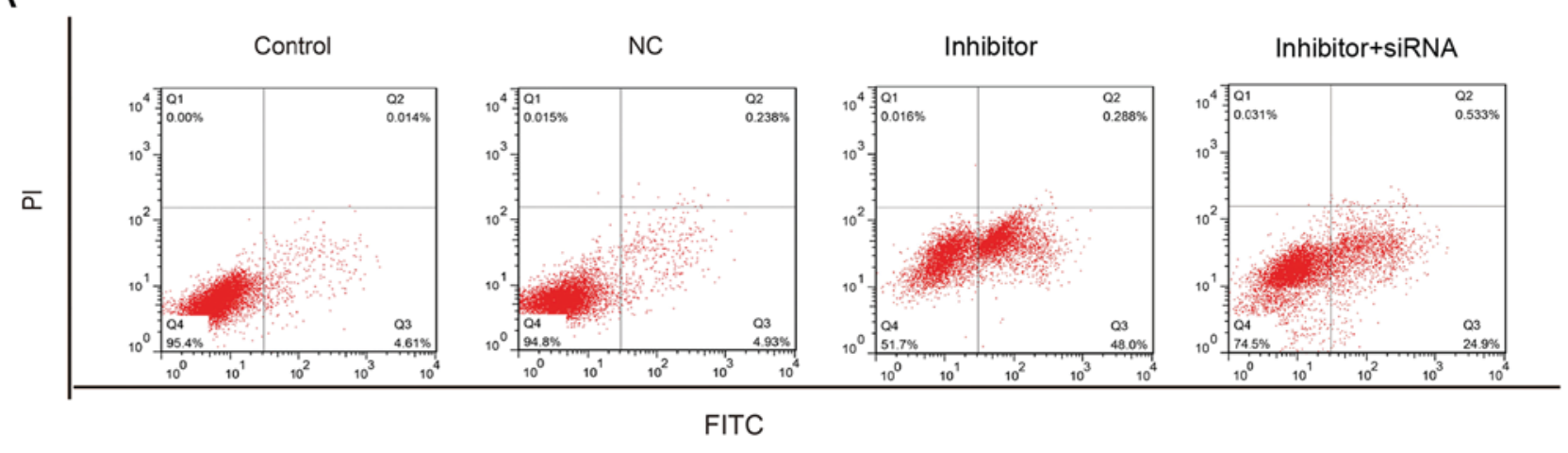

B

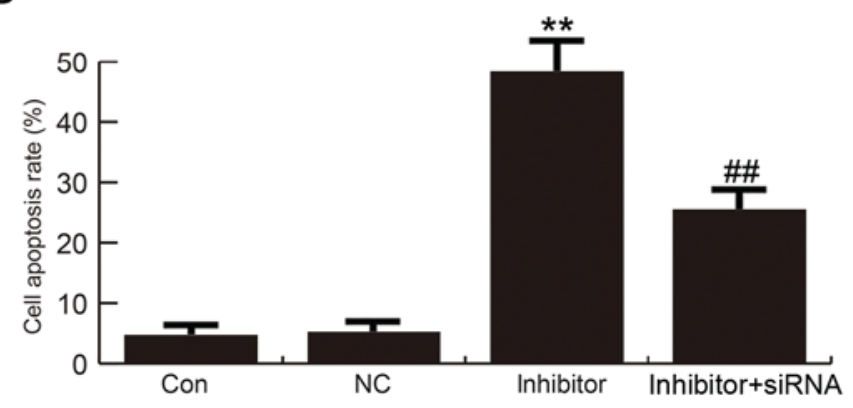

Figure 5. MicroRNA-663b inhibitor induces the apoptosis of T24 cells. (A) Flow cytometry was used to detect the cell apoptosis, and (B) data was quantified. Q1, dead cells; Q2, late apoptosis cells; Q3, early apoptosis cells; Q4, survival cells. Cell apoptosis = early apoptosis + late apoptosis. All data are presented as the mean \pm standard deviation of three independent experiments. ${ }^{* *} \mathrm{P}<0.01$ vs. control; ${ }^{\# \#} \mathrm{P}<0.01$ vs. inhibitor. Con, control group, no treatment; $\mathrm{NC}$, negative control inhibitor; siRNA, TUSC2-small interfering RNA.

A

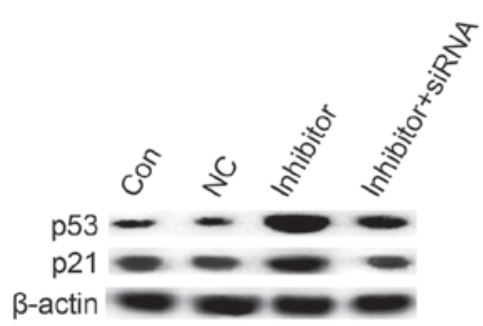

B

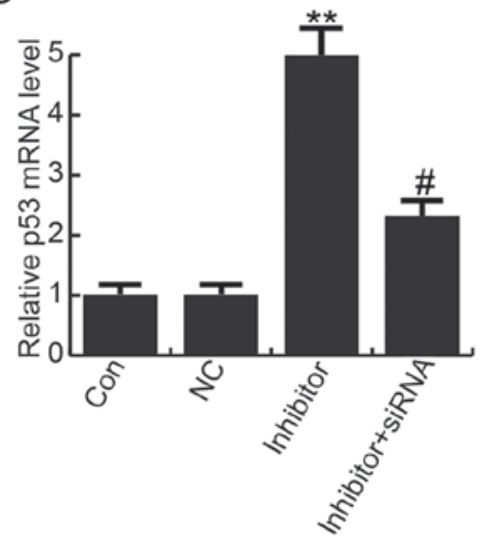

C

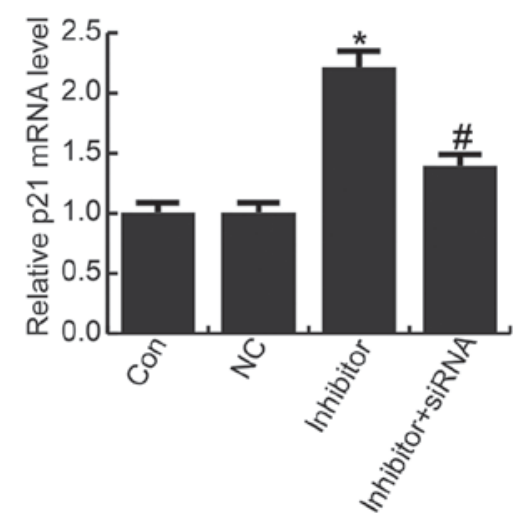

Figure 6. Effects of miR-663b inhibitor and TUSC2-siRNA on p53 and p21 expression. (A) Protein levels of p53 and p21 in T24 cells. Relative mRNA levels of (B) p53 and (C) p21 in T24 cells. All data are presented as the mean \pm standard deviation of three independent experiments. ${ }^{*} \mathrm{P}<0.05$ vs. inhibitor; "P $<0.05$ and ${ }^{* *} \mathrm{P}<0.01$ vs. control. TUSC2, tumor suppressor candidate 2; Con, control group, no treatment; NC, negative control inhibitor; siRNA, TUSC2-small interfering RNA.

bladder cancer more convincing. For instance, the association of miR-663b expression with the clinical characteristics and prognosis of patients with bladder cancer required evaluation. Additionally, whether miR-663b has a similar effect on bladder cancer in vivo requires further investigation. Only one bladder cancer line was used in the present study, the effect of miR-663b needs to be established in more bladder cancer cell lines. Furthermore, the effects of TUSC 2 and miR-663b overexpression in bladder cancer were not determined.
Furthermore, the in vitro analysis of bladder cancer is considerably different from the bladder cancer in patients, thus further in vivo experiments and clinical studies are required to validate the role of miR-663b in bladder cancer. In-depth research on these issues will be performed in the future.

\section{Acknowledgements}

Not applicable. 


\section{Funding}

No funding was received.

\section{Availability of data and materials}

The datasets used and/or analyzed during the current study are available from the corresponding author on reasonable request.

\section{Authors' contributions}

JC contributed to the design of the study, data collection, statistical analysis and data interpretation. BS contributed to data collection, manuscript preparation and literature search. GK contributed to the design of the study and to the preparation of the manuscript.

\section{Ethics approval and consent to participate}

The study was approved by The Ethics Committee of Beijing Luhe Hospital (Beijing, China).

\section{Patient consent for publication}

All patients provided their informed consent.

\section{Competing interests}

The authors declare that they have no competing interests.

\section{References}

1. Swarup V and Rajeswari MR: Circulating (cell-free) nucleic acids-a promising, non-invasive tool for early detection of several human diseases. FEBS Lett 581: 795-799, 2007.

2. Antoni S, Ferlay J, Soerjomataram I, Znaor A, Jemal A and Bray F: Bladder cancer incidence and mortality: A global overview and recent trends. Eur Urol 71: 96-108, 2017.

3. Moyer VA and U.S. Preventive Services Task Force: Screening for bladder cancer: U.S. Preventive Services Task Force recommendation statement. Ann Intern Med 155: 246-251, 2011.

4. Griffiths TR and Action on Bladder Cancer: Current perspectives in bladder cancer management. Int J Clin Pract 67: 435-448, 2013

5. Smith AB, Crowell K, Woods ME, Wallen EM, Pruthi RS, Nielsen ME and Lee CT: Functional outcomes following radical cystectomy in women with bladder cancer: A systematic review. Eur Urol Focus 3: 136-143, 2017.

6. Schulz GB, Grimm T, Buchner A, Jokisch F, Grabbert M, Schneevoigt BS, Kretschmer A, Stief CG and Karl A: Prognostic value of the preoperative platelet-to-leukocyte ratio for oncologic outcomes in patients undergoing radical cystectomy for bladder cancer. Clin Genitourin Cancer 15: e915-e921, 2017.

7. Coleman EA, Boult C and American Geriatrics Society Health Care Systems Committee: Improving the quality of transitional care for persons with complex care need. J Am Geriatr Soc 51: 556-557, 2003

8. Ambros V: microRNAs: Tiny regulators with great potential. Cell 107: 823-826, 2001

9. Bartel DP: MicroRNAs: Genomics, biogenesis, mechanism, and function. Cell 116: 281-297, 2004.

10. Valencia-Sanchez MA, Liu J, Hannon GJ and Parker R: Control of translation and mRNA degradation by miRNAs and siRNAs. Genes Dev 20: 515-524, 2006.

11. Croce CM: Causes and consequences of microRNA dysregulation in cancer. Nat Rev Genet 10: 704-714, 2009.

12. Catto JW, Alcaraz A, Bjartell AS, De Vere White R, Evans CP, Fussel S, Hamdy FC, Kallioniemi O, Mengual L, Schlomm T and Visakorpi T: MicroRNA in prostate, bladder, and kidney cancer: A systematic review. Eur Urol 59: 671-681, 2011.
13. Shi Z, Wei Q, Zhang M and She J: MicroRNAs in bladder cancer: Expression profiles, biological functions, regulation, and clinical implications. Crit Rev Eukaryot Gene Expr 24: 55-75, 2014.

14. Gottardo F, Liu CG, Ferracin M, Calin GA, Fassan M, Bassi P, Sevignani C, Byrne D, Negrini M, Pagano F, et al: Micro-RNA profiling in kidney and bladder cancers. Urol Oncol 25: 387-392, 2007.

15. Du M, Shi D, Yuan L, Li P, Chu H, Qin C, Yin C, Zhang Z and Wang M: Circulating miR-497 and miR-663b in plasma are potential novel biomarkers for bladder cancer. Sci Rep 5: 10437, 2015.

16. Xin J, Zhang XK, Xin DY, Li XF, Sun DK, Ma YY and Tian LQ: FUS1 acts as a tumor-suppressor gene by upregulating miR-197 in human glioblastoma. Oncol Rep 34: 868-876, 2015.

17. Uzhachenko R, Shanker A, Yarbrough WG and Ivanova AV: Mitochondria, calcium, and tumor suppressor Fus1: At the crossroad of cancer, inflammation, and autoimmunity. Oncotarget 6: 20754-20772, 2015.

18. Li L, Yu C, Ren J, Ye S, Ou W, Wang Y, Yang W, Zhong G, Chen X, Shi H, et al: Synergistic effects of eukaryotic coexpression plasmid carrying LKB1 and FUS1 genes on lung cancer in vitro and in vivo. J Cancer Res Clin Oncol 140: 895-907, 2014.

19. Uzhachenko R, Ivanov SV, Yarbrough WG, Shanker A, Medzhitov R and Ivanova AV: Fus1/Tusc2 is a novel regulator of mitochondrial calcium handling, $\mathrm{Ca} 2+$-coupled mitochondrial processes, and $\mathrm{Ca} 2+-$ dependent NFAT and NF- $\kappa \mathrm{B}$ pathways in CD4+ T cells. Antioxid Redox Signal 20: 1533-1547, 2014.

20. Lin J, Sun T, Ji L, Deng W, Roth J, Minna J and Arlinghaus R: Oncogenic activation of c-Abl in non-small cell lung cancer cells lacking FUS1 expression: Inhibition of c-Abl by the tumor suppressor gene product Fus1. Oncogene 26: 6989-6996, 2007.

21. Ivanova AV, Ivanov SV, Prudkin L, Nonaka D, Liu Z, Tsao A, Wistuba I, Roth J and Pass HI: Mechanisms of FUS1/TUSC2 deficiency in mesothelioma and its tumorigenic transcriptional effects. Mol Cancer 8: 91, 2009.

22. Rimkus T, Sirkisoon S, Harrison A and Lo HW: Tumor suppressor candidate 2 (TUSC2, FUS-1) and human cancers. Discov Med 23: 325-330, 2017.

23. Livak KJ and Schmittgen TD: Analysis of relative gene expression data using real-time quantitative PCR and the 2(-Delta Delta C(T)) method. Methods 25: 402-408, 2001.

24. Shu Y, Ye W, Gu YL and Sun P: Blockade of miR-663b inhibits cell proliferation and induces apoptosis in osteosarcoma via regulating TP73 expression. Bratisl Lek Listy 119: 41-46, 2018.

25. Rogers $K$ and Chen X: Biogenesis, turnover, and mode of action of plant microRNAs. Plant Cell 25: 2383-2399, 2013.

26. Cho WC: MicroRNAs: Potential biomarkers for cancer diagnosis, prognosis and targets for therapy. Int J Biochem Cell Biol 42: 1273-1281, 2010

27. Yang X, Shi L, Yi C, Yang Y, Chang L and Song D: MiR-210-3p inhibits the tumor growth and metastasis of bladder cancer via targeting fibroblast growth factor receptor-like 1. Am J Cancer Res 7: 1738-1753, 2017.

28. Luo H, Yang R, Li C, Tong Y, Fan L, Liu X and Xu C MicroRNA-139-5p inhibits bladder cancer proliferation and self-renewal by targeting the Bmil oncogene. Tumour Biol 39: 1010428317718414, 2017.

29. Wang W, Shen F, Wang C, Lu W, Wei J, Shang A and Wang C: MiR-1-3p inhibits the proliferation and invasion of bladder cancer cells by suppressing CCL2 expression. Tumour Biol 39: $1010428317698383,2017$.

30. Wu D, Niu X, Tao J, Li P, Lu Q, Xu A, Chen W and Wang Z: MicroRNA-379-5p plays a tumor-suppressive role in human bladder cancer growth and metastasis by directly targeting MDM2. Oncol Rep 37: 3502-3508, 2017.

31. Takai T, Yoshikawa Y, Inamoto T, Minami K, Taniguchi K, Sugito N, Kuranaga Y, Shinohara H,Kumazaki M, Tsujino T, et al: A novel combination rnai toward warburg effect by replacement with miR-145 and silencing of PTBP1 induces apoptotic cell death in bladder cancer cells. Int J Mol Sci 18: E179, 2017.

32. Meng J, Majidi M, Fang B, Ji L, Bekele BN, Minna JD and Roth JA: The tumor suppressor gene TUSC2 (FUS1) sensitizes NSCLC to the AKT inhibitor MK2206 in LKB1-dependent manner. PLoS One 8: e77067, 2013.

33. da Costa Prando E, Cavalli LR and Rainho CA: Evidence of epigenetic regulation of the tumor suppressor gene cluster flanking RASSF1 in breast cancer cell lines. Epigenetics 6: $1413-1424,2011$ 